\title{
Minimal Walking Technicolor Simulation using Generative Adversarial Networks (GANs)
}

\author{
Essam Al Daoud \\ Computer Science Department, Zarqa University, Zarqa, Jordan, essamdz@ zu.edu.jo
}

\begin{abstract}
The Minimal Walking Technicolor (MWT) has been studied extensively and it was an excellent candidate for breaking the electroweak symmetry. However, to verify the correctness of the Lagrangian of the minimal walking Technicolor, the theoretical simulation of the Feynman rules that are extracted from Lagrangian of MWT must be identical to the data accumulated from practical experiments that are done by the large hadron collider (LHC). In this paper we amplified the rare events by generating samples similar to the original distribution usinga generative adversarial network (GAN). The visualization of the results indicates that the minimal walking Technicolor could be the best model to interpret the ambiguous phenomena.
\end{abstract}

Key words: Technicolor, Lagrangian, Simulation, Generative Adversarial Network

\section{INTRODUCTION}

A generative adversarial network (GAN) is a new architecture suggested by Goodfellow [1].The main task of the generative adversarial network is to generate new samples that come from an existing distribution of a given data. GAN consists from two neural network models. The first model is called a generator which is used to generate new samples similar to the expected one. This model can be used to generate fake copies similar to the original one. The second model is the discriminator. The goal of the discriminator is to classify the input vectors to real or not real. Thus, a discriminator tries to detect the fake copies. Therefore, there is a competition between the two models the discriminator tries to exclude the samples not fit in the given distribution and the generator tries to learn how to generate new sample fits in the given distribution. After training, the generative model can create new samples on demand. Figure1 illustrates GAN backpropagation processes.

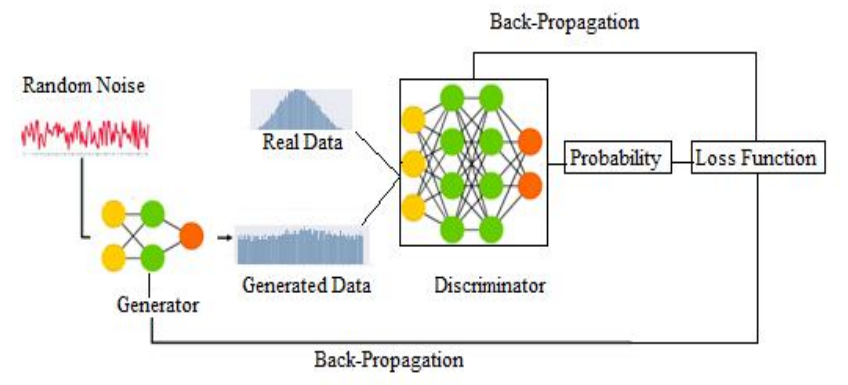

Figure 1:. A generative adversarial network (GAN).

GANcan be used to solve several important problems, for example: generate new samples for imbalanced datasets and rare classes, generate human faces or human poses that has super resolution, image-to-image translation, clothing translation, video prediction and so on. Since GAN were proposed, several designs have suggested that are built on the first architecture such as DcGan, StyleGan, BigGan, CycleGAN and CGAN [2]. Deep convolutional generative adversarial network (DcGan) integrates an unsupervised deep learning framework with convolutional networks. DCGAN shows very impressive results. The major changes, including the use of batch normalization for both the discriminator and the generator, the replacement of pooling layers and the replacement of fully connected hidden layers. StyleGanis introduced to produce authentic-looking images and to offer more control over image features. For example, the last layers determine micro-features, the middle layers determine hairstyle and facial expressions, and the first layers determine general outline and pose. The architecture of StyleGan consists of several layers and eight fully connected layers. BigGan achieves high inception score and creates realistic hair, skin and texture, however the disadvantages of BigGan is the need of high computation power. CycleGANis very useful to image-to-image translation and similar applications. CycleGAN tries to minimize the cyclic loss when translating an image to another and vice versa. Conditional GAN (CGAN) gives the user the highest control using conditions on auxiliary information, parameters to generate data and labels with the input data [3].

\section{MINIMAL WALKING TECHNICOLOR}

Lagrangian formulation is the most elegant and easiest way to present the modern elementary particles model. It captures the 
system dynamics and can be derived from potential and kinetic energy. However, it must has several properties such as: the total probability is conserved, invariant under certain symmetry groups, translational invariance and naturalness [4-6]. Lagrangian of the standard model (SM) can be described by equations (1)-(11), which contain four types of interactions: the electromagnetic interactions, the strong interactions, Yukawa interactions and weak interactions. All the interactions are mediated by bosons such as gluons, photons, $\mathrm{W}+, \mathrm{W}-$, and $\mathrm{Z}$

$$
\begin{gathered}
\mathcal{L}=\mathcal{L}_{\text {fermioni }}+\mathcal{L}_{\text {gauge }}+\mathcal{L}_{\text {Yukawa }}+\mathcal{L}_{\text {Higgs }} \\
\mathcal{L}_{\text {gauge }}=-\frac{1}{4} B_{\mu \nu} B^{\mu \nu}-\frac{1}{4} \bar{W}_{\mu \nu} \bar{W}^{\mu \nu} \\
\mathcal{L}_{\text {fermioni }}=\mathcal{L}_{\text {quark }}+\mathcal{L}_{\text {lept }} \\
\mathcal{L}_{\text {Higgs }}=-V(\phi)+\frac{1}{2}\left(D_{\mu} \phi\right)^{2} \\
\mathcal{L}_{\text {Yukawa }}=-G_{i}\left(\bar{R} \phi^{T^{*}} L+\bar{L} \phi R\right) \\
V=b \phi^{4}+a \phi^{2} \\
\phi=\left(\begin{array}{l}
\phi_{1} \\
\phi_{2}
\end{array}\right) \\
\mathcal{L}_{\text {lept }}=\bar{\ell}_{R} \bar{\gamma}_{\mu} G_{\mu} \ell_{R}+\sum_{l=e, u, \tau}(\bar{v}, \bar{\ell})_{L} \gamma D_{\mu}\left(\begin{array}{l}
v \\
\ell
\end{array}\right)_{L} \\
\mathcal{L}_{\text {quark }}=X^{X}+\bar{u}_{R} \gamma_{\mu} G_{\mu} u_{R}+\bar{d}_{R} \gamma_{\mu} G_{\mu} d_{R} \\
D_{\mu}=\partial_{\mu}-i g{ }^{\prime} B_{\mu}+i \frac{g}{2} \vec{\sigma} \vec{W} \\
G_{\mu}=-i g \frac{Y}{2} B_{\mu}+\partial_{\mu}
\end{gathered}
$$

Despite the success of the standard theory, it is imperfect model. There are some phenomena, experimental results and theoretical conclusions are not clarified by the standard theory, namely, dark energy, dark matter, hierarchy problem, proton radius puzzle, neutrino masses and gravity [7]. Therefore, several models that enhance the standard model and consistent with these phenomena have been suggested. New models such as: Minimal Walking Technicolor (MWT), two-Higgs-Doublet Model (2HDM), Minimal Supersymmetric Standard Model (MSSM) and Higgs Effective Field Theory (HEFT) [7].

Minimal Walking Technicolor (MWT) is introduced for more complete model and to solve some famous problems including gauge coupling unification and dark matter. MWT consists of two adjoint Dirac technifermions and an SU (2) technicolor. The fermions can be written as [8]:

$$
Q^{a}=\left(\begin{array}{c}
U_{L}^{a} \\
D_{L}^{a} \\
-i \sigma^{2} U_{R}^{* a} \\
-i \sigma^{2} D_{R}^{* a}
\end{array}\right), \mathrm{a}=1,2,3 .
$$

The general symmetry SU(4) could be break to the subgroup $\mathrm{SO}(4)$ by the following equation:

$$
\left\langle Q_{i}^{\alpha} Q_{j}^{\beta} \epsilon_{\alpha \beta} E^{i j}\right\rangle=-2\left\langle\overline{U_{R}} U_{L}+\overline{D_{R}} D_{L}\right\rangle
$$

Feynman rules and the scattering amplitudes can be found by Lagrangian of a model. Therefore, the package FeynRuleshas been used to construct the Feynman rules for any Lagrangian[9-11]. However, all the particles defined by the Lagrangian must be specified clearly. The next step is to use the generative adversarial network (GAN) to solve the skew of the output data [12-14].

\section{NEW DATA GENERATION}

A generative adversarial network is belong to generative models, which considered a subset of unsupervised learning, the generative models have been used to learn the underlying structure of a dataset and to discover the distribution function of the input data $p(x)$ allowing them to generate new samples. There are several famous generative models such as real NVP, pixel-CNN, pixel-RNN and variational auto-encoders (VAEs). Each model has its own advantages. However, GAN have proven to be the best with very high dimensional data, it can generate the sharpest images and can model $p(x)$ without explicitly defining the distribution function. On another hand, due to unstable training dynamics, GANs are difficult to optimize and no statistical reasoning can be done with them. Figure () shows two neural network models, the first is the generator $G(\boldsymbol{z}, \boldsymbol{\theta} \square)$.The role of the generator is mapping an input variables $z$ to the output space $\boldsymbol{x}$. Conversely, the discriminator $D(\boldsymbol{x}, \boldsymbol{\theta} \square)$ maps an input $\mathrm{x}$ to the range $(0,1)$ which represents the probability of $x$ to be fake or real. $\theta_{i}$ represents the neural networks weights of the both cases. Figure 2 shows new data generation by GAN.

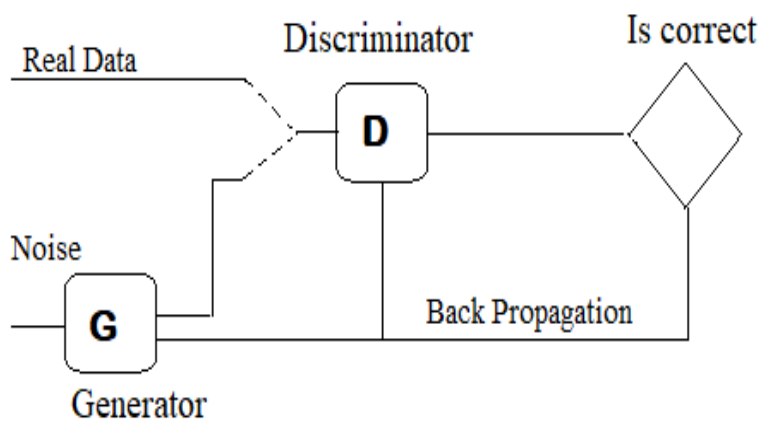

Figure 2: New data generation by GAN.

The discriminator is trained to classify the input correctly, the back-propagation is applied to minimizes $D(G(z))$ and maximizes the function $D(x)$, while the generator is trained to fool the discriminator, the back-propagation is applied to maximizes $D(G(z))$ using the loss function. After several steps of training, if there is no any further improvement and the discriminator could not differentiate between fake and real inputs, then the generator can be used to generate realistic 
Essam Al Daoud, International Journal of Emerging Trends in Engineering Research, 8(3), March 2020, 759 - 762

synthetic data. Equation (14) shows the core process during the training [3].

$\min _{G} \max _{D} V(D, G)=$

$\mathbb{E} x \sim$ pdataxlog $D x+\mathbb{E} z \sim$ pzxzlog $1-D G(z)$

The following steps can be used to train the generative adversarial network using stochastic gradient descent

1. Sample a real-data set and a noise set, with size $n$.

2. Use real and fake data to train the Discriminator:

$$
\nabla_{D} \sum\left(\log D\left(x^{i}\right)\right)+\log \left(1-D\left(G\left(z^{i}\right)\right)\right)
$$

3. Use noise set with size $n$ to train the Generator:

$$
\nabla_{G} \sum \log \left(1-D\left(G\left(z^{i}\right)\right)\right)
$$

\section{Repeat from Step 1.}

\section{SIMULATION RESULTS}

The first step is to amplify the rare data using the generator that is trained using the previous algorithm. Figure 3 shows the loss function for the discriminator and generator, where the both become very close to each other.

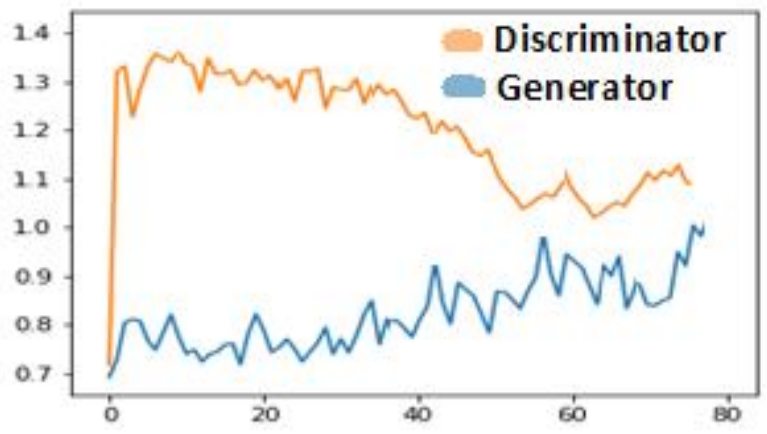

Figure 3: The loss function for the discriminator and generator.

Four models are studied and compared: Minimal Walking Technicolor (MWT), The standard model (SM), minimal supersymmetric standard model (MSSM) and two-Higgs doublet model (2HDM), MadGraph 5 is used to simulate the detectors, generative adversarial network (GAN) is used to solve the imbalanced problem in the data, while MadAnalysis 5 is used to interpret the results and draw the curves. Table 1 and 2 show the number of diagrams and processes generated by the models.
Table 1: Diagrams For Each Collision

\begin{tabular}{|c|c|c|c|c|}
\hline Collision & MWT & MSSM & SM & 2HDM \\
\hline pp to all all & 480 & 501 & 126 & 4058 \\
\hline pp to a a & 8 & 8 & 8 & 8 \\
\hline pp to j jj & 781 & 781 & 781 & 7565 \\
\hline pp to leptlept & 28 & 28 & 28 & 124 \\
\hline pp to $1+1-$ & 16 & 16 & 16 & 112 \\
\hline pp to $1+1+1-1-$ & 490 & 480 & 480 & 34512 \\
\hline p to all all & 144 & 152 & 39 & 155 \\
\hline
\end{tabular}

Table 2: Processes For Each Collision.

\begin{tabular}{|c|c|c|c|c|}
\hline Collision & MWT & MSSM & SM & 2HDM \\
\hline Pp to all all & 290 & 304 & 75 & 935 \\
\hline pp to a a & 4 & 4 & 4 & 4 \\
\hline pp to j jj & 97 & 97 & 97 & 293 \\
\hline pp to leptlept $~$ & 20 & 20 & 20 & 44 \\
\hline pp to l+1- & 8 & 8 & 8 & 16 \\
\hline pp to l+ l+ 1- 1- & 13 & 12 & 12 & 72 \\
\hline p to all all & 140 & 152 & 39 & 143 \\
\hline
\end{tabular}

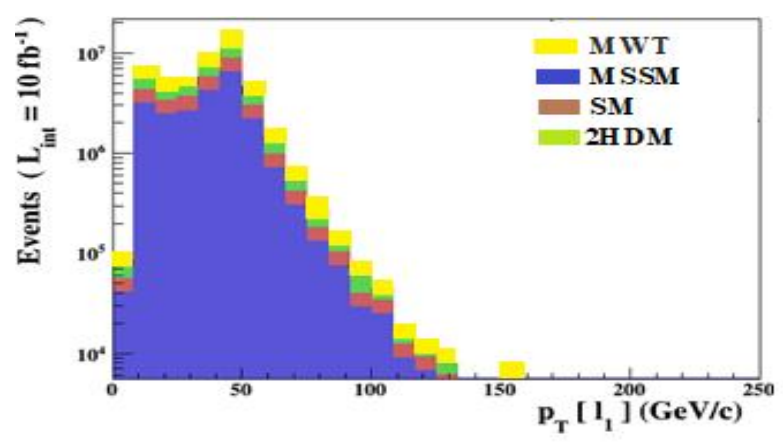

Figure 4:. The distribution of the leading lepton

To compare the models, $13 \mathrm{TeV}$ energy is simulated for each experiment and 10000 events are generated. Figure 4 shows the mean of the momentum of the leading lepton, where the results indicate that there is a clear difference in the distribution of the tested modes.

\section{CONCLUSION}

A generative adversarial network is a very powerful tool to generate new samples fit into a given data distribution. GAN can be used to solve the imbalanced data or rare events generation. To find the optimal weights of GANs several iterations must be implemented. Thus, Nash equilibrium in non-convex, continuous and high dimensional must be found. In particle physics, there are some phenomena cannot be explained using the traditional models, minimal walking technicolor is introduced to expand the stranded model, however, to solve the rare events problem GAN is used. The visualization of the results indicates that the minimal walking Technicolor could be the best model to interpret the ambiguous phenomena. 


\section{ACKNOWLEDGMENT}

This research is funded by the Deanship of Research and Graduate Studies in Zarqa University /Jordan

\section{REFERENCES}

[1] Goodfellow, Ian J and Pouget-Abadie et al, "Generative adversarial networks", ArXiv e-prints (2014) [1406.2661].

[2] A. Odena, Semi-Supervised Learning with Generative Adversarial Networks, ArXiv e-prints (June, 2016) [1606.01583].

[3] Salimans, T., Goodfellow, I., Zaremba, W., Cheung, V., Radford, A., and Chen, X. (2016). Improved techniques for training gans. In the book "In Advances in Neural Information Processing Systems", pages 2226-2234.

[4] J. P. Guillet, G. Heinrich, and J. F. von Soden-Fraunhofen, "Tools for NLO automation: extension of the golem95C integral library," Comput. Phys. Commun.Vol. 185, pp 1828-1834, 2014. https://doi.org/10.1016/j.cpc.2014.03.009

[5] J. Alwall et al., "The automated computation of treelevel and next-toleading order differential cross sections, and their matching to parton shower simulations", JHEP 1407, arXiv:1405.0301, 2014.

[6] C. Degrades et al., "UFO-The Universal FeynRules Output," Comput. Phys. Commun., vol. 183, pp 12011214, 2012.

[7] J. Alwall, M. Herquet, F. Maltoni, O. Mattelaer, and T. Stelzer, "MadGraph 5: Going Beyond," JHEP 1106, arXiv:1106.0522, 2011. https://doi.org/10.1007/JHEP06(2011)128

[8] J. T. Childers, T. D. Uram, T. J. LeCompte, M. E. Papka and D. P. Benjamin, Simulation of LHC events on a millions threads, J. Phys. Conf. Ser. 664 (2015), no. 9 092006.

[9] T. Peraro, "Ninja: Automated Integrand Reduction via Laurent Expansion for One-Loop Amplitudes," Comput. Phys. Commun. vol. 185, pp 2771-2797, 2014.

[10]E. Al Daoud, "An Efficient Algorithm for Finding a Fuzzy Rough Set Reduct Using an Improved Harmony Search," I.J. Modern Education and Computer Science, vol. 7, no. 2, pp16-23, 2015.

https://doi.org/10.5815/ijmecs.2015.02.03

[11]E. Al Daoud, H Turabieh, "New empirical nonparametric kernels for support vector machine classification," Applied Soft Computing, vol. 13, no. 4, 1759-1765, 2013.

[12]L. Torrizo and A. Africa, "Next-hour electrical load forecasting using an artificial neural network: Applicability in the Philippines." International Journal of Advanced Trends in Computer Science and Engineering. Vol. 8, No. 3, pp. 831-835, 2019.

https://doi.org/10.30534/ijatcse/2019/77832019.

[13] P. M.V.D, "Human activity recognition using Deep Learning," International Journal of Emerging Trends in Engineering Research, vol. 7, no. 11, pp. 536-541, 2019. https://doi.org/10.30534/ijeter/2019/227112019

[14] A. Africa and C. Charleston Franklin, "Development of a cost-efficient waste bin management system with mobile monitoring and tracking." International Journal of Advanced Trends in Computer Science and Engineering. Vol. 8, No. 2, pp. 319-327, 2019. https://doi.org/10.30534/ijatcse/2019/35822019 